\title{
FDTD Study on Evolution of Trimer Silver@Silica Nanospheres to Dimer for SERS Characteristics
}

\author{
Anitharaj Nagarajan ${ }^{1} \cdot$ Aruna Priya Panchanathan ${ }^{1}$ (D) Pandian Chelliah $^{2} \cdot$ Hiroaki Satoh $^{3} \cdot$ Hiroshi Inokawa $^{3}$
}

Received: 17 May 2021 / Accepted: 4 October 2021 / Published online: 13 October 2021

() The Author(s), under exclusive licence to Springer Science+Business Media, LLC, part of Springer Nature 2021

\begin{abstract}
Light enhancement occurs strongly within the plasmonic clusters by interaction with surface plasmons. Surface-enhanced Raman spectroscopic (SERS) characteristics of a series of silver@ silica trimer core-shell (CS) nanosphere (NS) clusters are investigated in this paper. It is significant to understand the electric field (EF) enhancement mechanism behind the SERS technique. The effect of symmetry breaking is studied for the series starting from the highly symmetric trimer cluster and transformed to linear dimer geometry which progresses through the gradual reduction in the vertex NS. The optical activity such as the evolution of LSPR peak is discussed, the formation of hot spots is demonstrated and the strength of the local EF enhancement is calculated and correlated with the plasmon dipolar modes by using plasmon hybridization theory to understand the underlying physical concepts.
\end{abstract}

Keywords Core-shell nanosphere $\cdot$ Trimer $\cdot$ Dimer $\cdot$ Plasmon hybridization theory $\cdot$ LSPR $\cdot$ Hot spots

\section{Introduction}

Localized surface plasmon resonance (LSPR) effects observed in metallic nanostructures due to the collective oscillations of conductive electrons under irradiation draw increasing interest in the applications such as solar cells, plasmonic waveguides, LSPR sensors and surface-enhanced Raman spectroscopy (SERS) [1-10]. In SERS technique, which is a more promising choice for COVID-19 detection than the time-consuming PCR, the significant challenge is to understand the interplay between the structure of the nanoparticle and its optical response [11]. SERS characteristics depend on the formation of hot spots, which are strong electric field (EF) confinement, occurring in the gap between the two individual nanospheres, direction of polarization and the strength of the EF $[12,13]$. The features of a highly sensitive

Aruna Priya Panchanathan

arunaprp@srmist.edu.in

1 Department of Electronics and Communication Engineering, SRM Institute of Science and Technology, Kattankulathur, Tamilnadu, India 603203

2 Department of Physics, R.K.M. Vivekananda College, Chennai, India 600004

3 Research Institute of Electronics, Shizuoka University, Hamamatsu, Shizuoka 432-8011, Japan and rapidly analyzing SERS substrate should produce an enhancement factor greater than $10^{5}$, it should be stable under the effect of oxidation and should not be degraded under ambient environment conditions [14].

Plasmonic molecules are small clusters constructed by the aggregation of individual metallic nanospheres in controlled manner [15]. Plasmonic clusters with metals encapsulated by a dielectric layer enable tuning of wavelength in the range and prevent the effect of oxidation and instability under ambient environment condition [10, 16]. Dimer with nearly touching nanospheres is the mostly studied plasmonic molecules, due to its capability to yield strong hot spots [17]. The complex cluster, trimer, is more interesting as they not only confine light in the gaps, but may open the window into numerous novel fascinating phenomena by breaking its symmetry [18-20].

In our previous work, the study on the optimization and relation between the SERS enhancement characteristics and structure of silver@ silica trimer cluster composed of nanospheres with equal sizes have been determined by symmetry breaking. The highly symmetric trimer arrangement is transformed to linear trimer by gently opening the vertex angle. The effect of polarization, evolution of LSPR peak and optimization for a highly sensitive SERS substrate have been analysed and correlated with the plasmon dipolar modes using plasmon hybridization theory for understanding 
the background mechanism. The role of shell such as tuning of wavelength and twofold enhancement has also been discussed [21].

The current work focuses on symmetry breaking of the equilateral trimer cluster through a different scenario. By gently reducing the vertex nanosphere size, the symmetric trimer is transformed to linear dimer. The SERS enhancement factor is directly proportional to the fourth power of the ratio of the EF intensity around the NS to the EF intensity of the incident light $\left(|E| /\left|E_{0}\right|\right)^{4}[22]$ and could be determined by evaluating enhancement factor of nanocluster $\left(\left(|E| /\left|E_{0}\right|\right)^{2}\right)$. For this current series, the local EF enhancement is calculated, the polarization selective LSPR evolution is discussed and the formation of hot spots is demonstrated and correlated with the plasmon dipolar modes.

\section{Electromagnetic Simulation}

The electromagnetic simulation for investigating the SERS characteristics is performed by 3D-FDTD method using the open-source software package called MEEP [23]. The size of the computational cell is kept as $480 \mathrm{~nm}^{3}$, the Gaussian source of central frequency, $0.9 \mathrm{PHz}$ and width, $1.2 \mathrm{PHz}$. The boundary condition for $x, y$ and $z$ directions is made by perfectly matched layers of thickness $50 \mathrm{~nm}$. The resolution of the computation is 15 cells/nm with the spatial step of $0.1 \mathrm{~nm}$. Lorentz-Drude expression from Rakic's work is used to model Ag [24]. The refractive index of the silica and air used is 1.45 and 1 , respectively. The FDTD simulation method is verified with the theoretical result by comparing the optical spectra of the monomer between the simulated and the Mie's theory.

The symmetric trimer of $D_{3 \mathrm{~h}}$ symmetry group consists of three nearly touching ( $g a p=2 \mathrm{~nm}$ ) CS NS of radius, $R_{\mathrm{CS}}=30 \mathrm{~nm}$ (radius of silver, core is $R_{\mathrm{C}}=25 \mathrm{~nm}$ and thickness of silica is $5 \mathrm{~nm}$, i.e. $R_{\mathrm{CS}} / R_{\mathrm{C}}=1.2$ ). The symmetry breaking is done by gently reducing the vertex radius, $R_{1}=30 \mathrm{~nm}$, with $R_{\mathrm{CS}} / R_{\mathrm{C}}$ maintained at 1.2 and the base radius, $R_{2}$, and gap size are constant at 30 and $2 \mathrm{~nm}$, respectively leading to the transformation of $C_{2 \mathrm{v}}$ symmetry. Further reduction in size $\left(R_{1}=0 \mathrm{~nm}\right)$ leads to vanishing of vertex NS and formation of linear dimer ( $D_{\infty \mathrm{h}}$ symmetry) (Fig. 1).

\section{Results and Discussion}

\section{Symmetric Trimer}

The local electric field enhancement of the trimer with $R_{1}=30,25,15$ and $0 \mathrm{~nm}$ is calculated under both transverse and longitudinal polarizations and the plasmonic spectra are shown in Fig. 2. The $D_{3 \mathrm{~h}}$ symmetry with $R_{1}=30 \mathrm{~nm}$ shows EF enhancement with degenerative nature at two peaks. This degeneracy of the $D_{3 \mathrm{~h}}$ symmetry could be discussed by exploring the structure of its plasmon mode. According to plasmon hybridization theory, $D_{3 \mathrm{~h}}$ group consists of six in-plane modes in which three are bonding and three are anti-bonding modes. The correlation table shown in Fig. 3 indicates three bonding dipolar modes of $D_{3 \mathrm{~h}}$ symmetry, two (a)
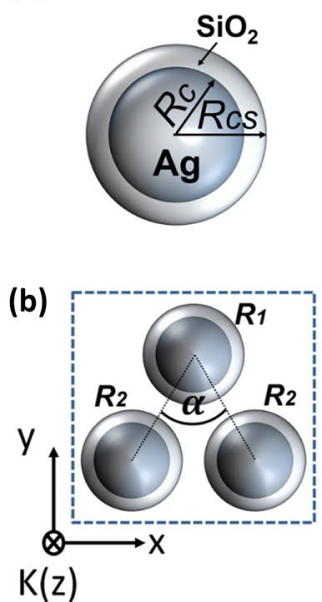

(c)

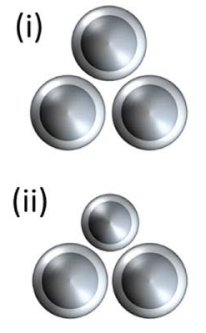

(iii)

(iv)

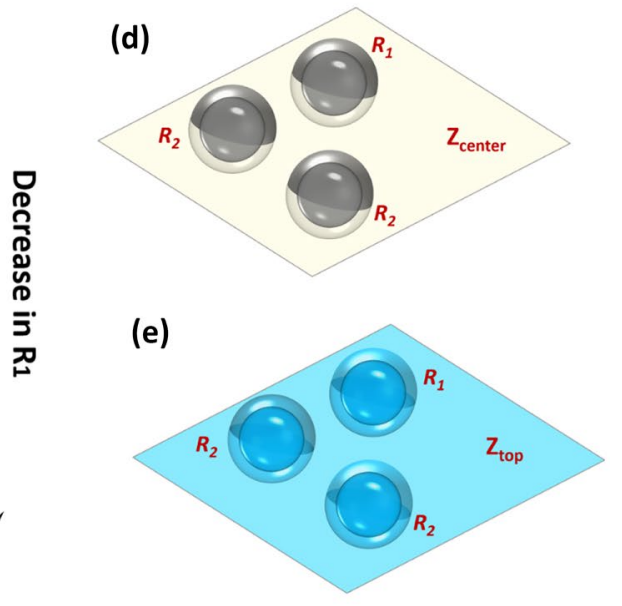

Fig. 1 (a) Schematic representation of core-shell monomer with core radius, $R_{\mathrm{C}}$, and core-shell radius, $R_{\mathrm{CS}}$; (b) Top view of the highly symmetric equilateral trimer cluster, with vertex angle, $\alpha$, vertex radius $=R_{1} \mathrm{~nm}$ and base radius $=R_{2} \mathrm{~nm}$. The local electric field enhancement factor is calculated at the $x y$ plane shown by the dashed square box; (c) Symmetry breaking by decreasing the size of the vertex radius, $R_{1}$ (i) $D_{3 \mathrm{~h}}$ symmetry, (ii) and (iii) $C_{2 \mathrm{v}}$ symmetry and (iv) $D_{\infty \mathrm{h}}$ symmetry; (d) $3 \mathrm{D}$ view of trimer with centre of vertex NS aligned at same $z$-position with the centre of base NSs, $Z_{\text {centre }}$ and (e) $3 \mathrm{D}$ view of trimer with top surface of three NSs touching at same $z$-position, $Z_{\text {top }}$ 


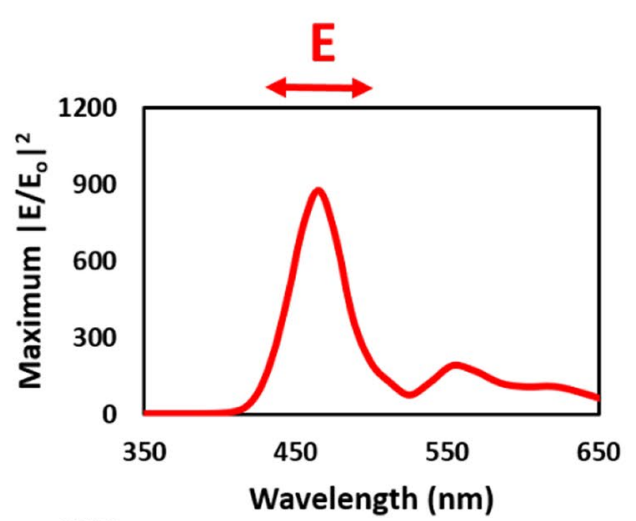

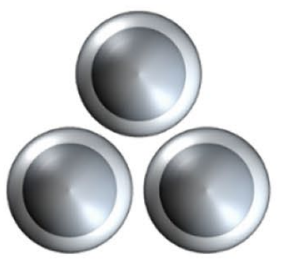

(a) $R_{1}=30 \mathrm{~nm}$
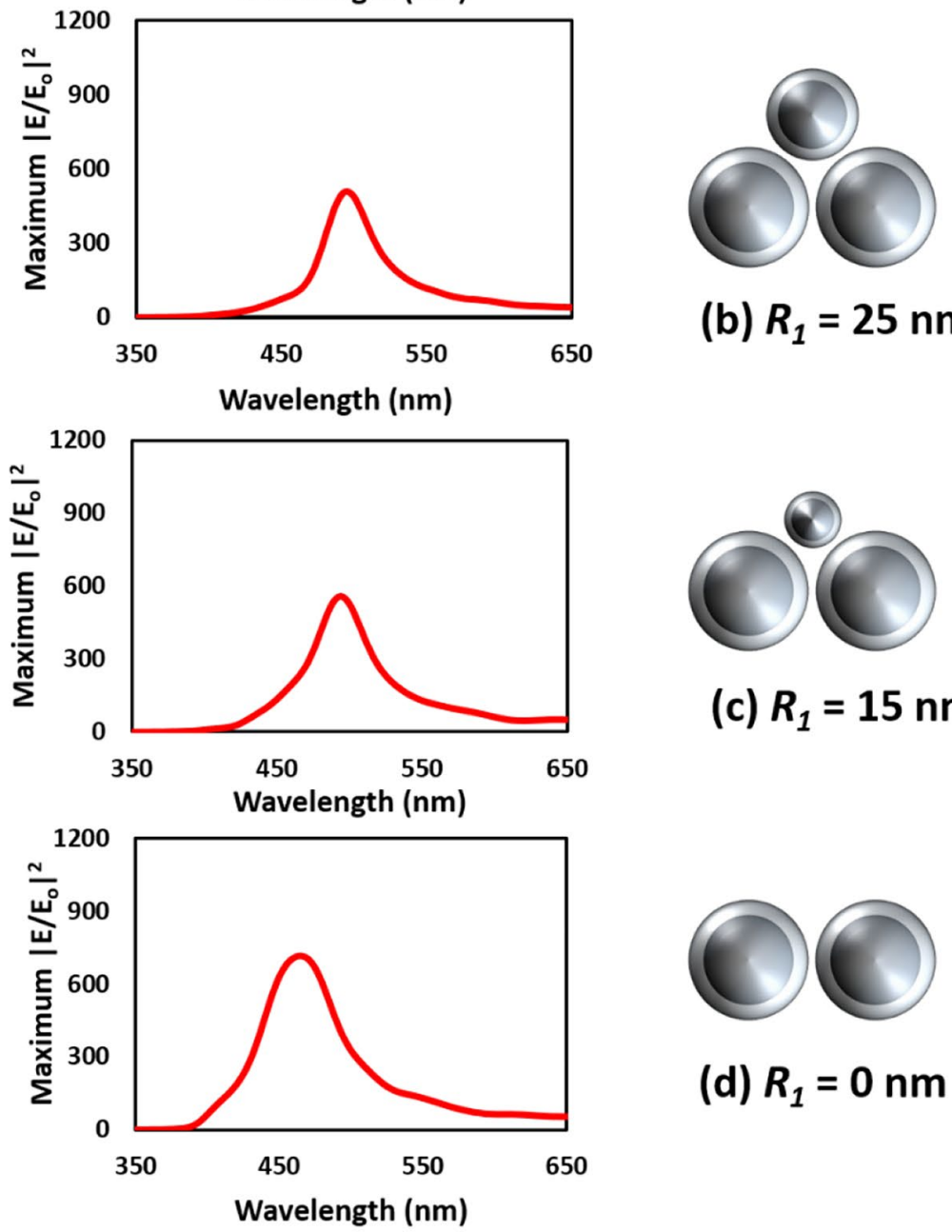

(d) $R_{1}=0 \mathrm{~nm}$

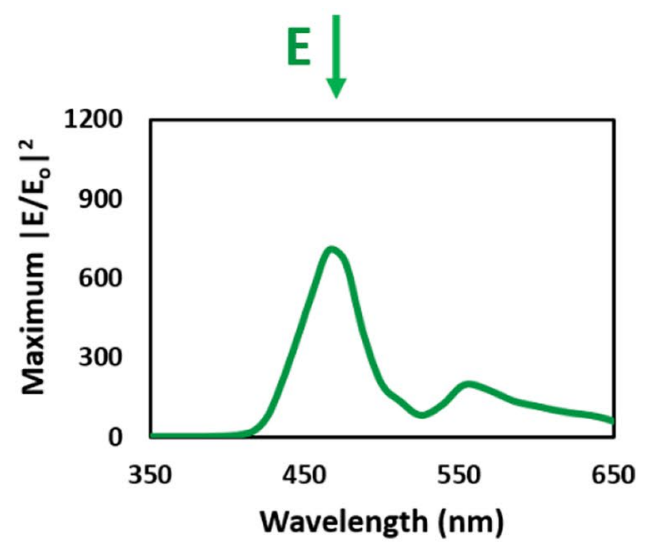

(b) $R_{1}=25 \mathrm{~nm}$

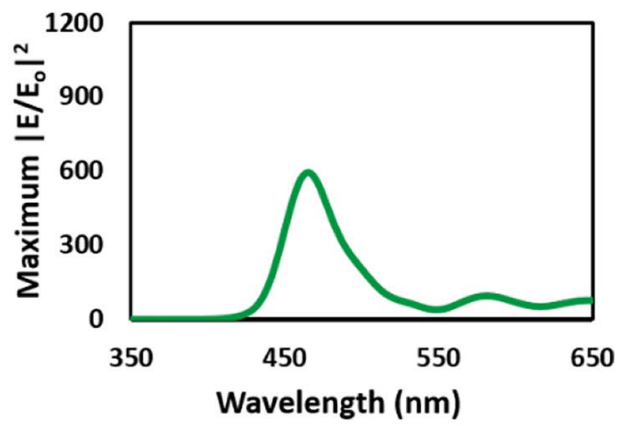

(c) $R_{1}=15 \mathrm{~nm}$
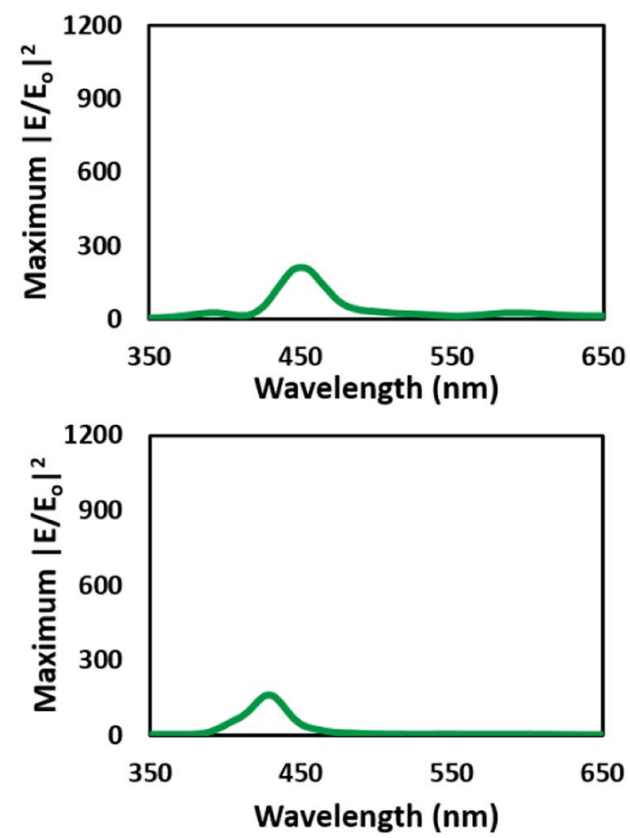

Fig. 2 The strength of local electric field enhancement for the trimer series with vertex angle, $\alpha$, vertex radius $=R_{1} \mathrm{~nm}$ and base radius $=R_{2} \mathrm{~nm}$. The series starts with $R_{1}=30 \mathrm{~nm}(\mathbf{a}), 25 \mathrm{~nm}(\mathbf{b}), 15 \mathrm{~nm}(\mathbf{c})$ and $0 \mathrm{~nm}(\mathbf{d})$ for transverse and longitudinal polarizations

$E^{\prime}$ and one $A_{2}^{\prime}$ modes in which the latter is dark and could be excited only by azimuthally polarized light. The high enhancement at $465 \mathrm{~nm}$ with almost same EF of 704 and 876 for transverse and longitudinal polarizations is assigned to two bonding $E^{\prime}$ modes as shown in Fig. 3. The small peak at $550 \mathrm{~nm}$ is corresponding to the two anti-bonding $E^{\prime}$ modes. Each set of bonding and anti-bonding $E^{\prime}$ modes has one mode with dipole arrangement in the longitudinal direction (longitudinal $E^{\prime}$ mode) while the other has its dipoles arranged in transverse direction (transverse $E^{\prime}$ mode). 


\section{Resizing the Vertex CS NS}

The resizing of vertex particle leads to the lift in the degeneracy of the plasmon modes and the corresponding transformation of the three bonding modes is clearly represented in correlation table for each trimer configuration. The polarization selective excitation of the mode and the associated electric field enhancement could be explained by using plasmon modes shown in Fig. 3. The $A_{1}$ mode of the $C_{2 \mathrm{v}}$ configuration is originated from the lift in the dipole orientation of transverse $E^{\prime}$ modes. For the case of $R_{1}=25$ and $15 \mathrm{~nm}$ under transverse polarization, the $A_{1}$ bonding mode gradually blue shifts and the electric field intensity slightly diminishes corresponding to the decrease in its dipole moment. When $R_{1}$ is reduced to $0 \mathrm{~nm}$, it corresponds to linear dimer of $D_{\infty \mathrm{h}}$ symmetry, the $A_{1}$ mode is transformed to the null mode of dimer cluster, the LSPR further blue shifts and electric field is decreased well. The e-field of null mode is similar to the intensity of the monomer, because all the localized charges are on the vertex CS NS with a zero size, while the charge amplitude of the base CS NSs is essentially zero.

On the other hand, under longitudinal polarization, for $C_{2 \mathrm{v}}$ configuration, the longitudinal $B_{2}$ bonding modes originated from $A_{2}{ }^{\prime}$ modes of $D_{3 \mathrm{~h}}$ symmetry are responsible for the strong peak in clusters with $R_{1}=25$ and $15 \mathrm{~nm}$, with interestingly no change in the position of the spectral peak. For $D_{\text {ch }}$ symmetry, transformation to bright $\Sigma_{u}^{+}$mode occurs which results in the stronger peak. The antibonding peak is gradually diminishes and vanishes in both the cases of transverse and longitudinal polarization, when the configuration

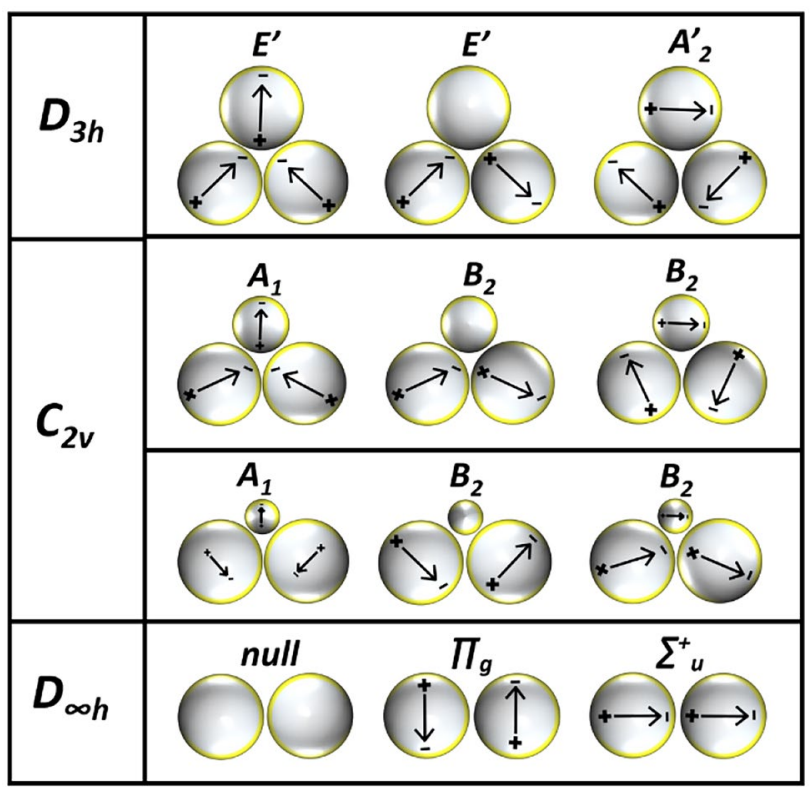

Fig. 3 Summary of correlation of bonding dipolar modes of trimer structure for decreasing sizes of vertex sphere of radius, $R_{1}$

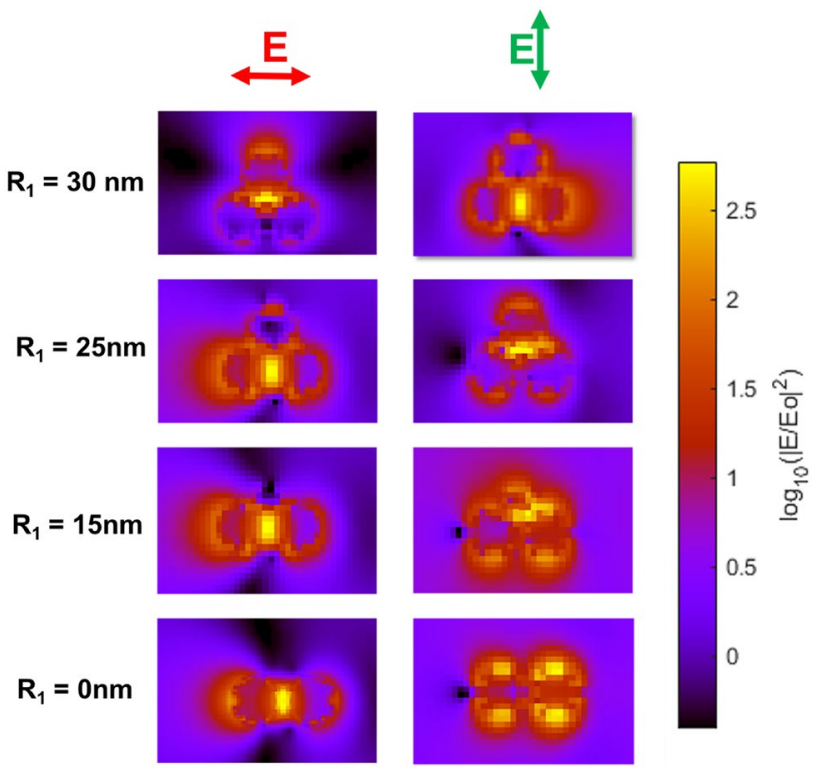

Fig. 4 Spatial map of the EF intensity for the series starting from equilateral trimer to linear dimer with decreasing $R_{1}$ from 30 to $0 \mathrm{~nm}$ under transverse and longitudinal polarizations. Note the generation of hot spots in the gap

is altered from $D_{3 \mathrm{~h}}$ symmetry to $D_{\infty \mathrm{h}}$ symmetry. The surface charge excitation is not enough to enhance the e-field and only a small bonding peak appears for $D_{\infty \mathrm{h}}$ symmetry, enabling polarization selective switching of e-field enhancement in dimers.

Figure 4 shows the plot of spatial distribution for CS NS trimers to demonstrate the generation of e-field hot spots for understanding the e-field localization in different trimer configurations. For longitudinal and transverse polarization in $D_{3 \mathrm{~h}}$ symmetry, hot spots rises at the gap between three NS corresponding to the longitudinal $E^{\prime}$ mode and gap between the base NS corresponding to the dipole orientation of transverse $E^{\prime}$ mode, respectively. As the degeneracy is lifted, e-field selectively diminishes with respect to the direction of polarization. In $C_{2 \mathrm{v}}$ symmetry, for longitudinal polarization, hot spot arises between the base NSs corresponding to the $B_{2}$ mode with decreases e-field intensity and for transverse polarization exhibits apparent diminishing of hot spots. In $D_{\infty \mathrm{h}}$ symmetry, hot spots are switched on and off corresponding to the bright $\Sigma_{u}^{+}$mode in longitudinal polarization and null mode in transverse polarization.

\section{Effect of Location of Centre of Vertex CS NS}

The electric field enhancement and LSPR mode of the trimer series are analysed at the $Z_{\text {centre }}$, i.e. same $z$-position of centre of the three CS NSs and discussed in the previous sections. The effect of the trimer series on the electric field enhancement and the LSPR wavelength with respect to the 
(a)

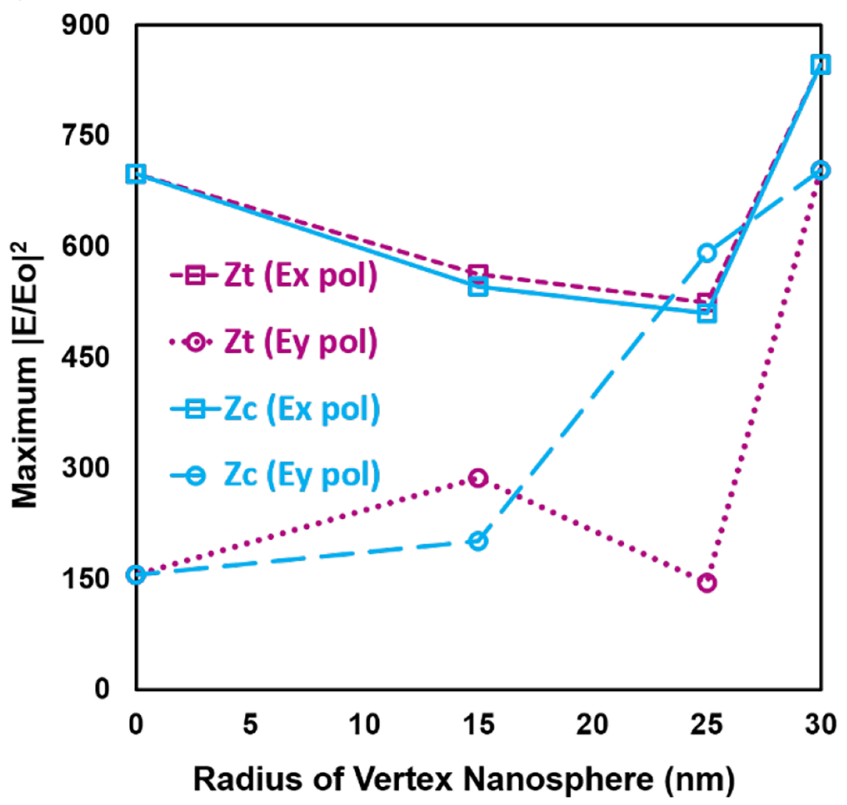

(b)

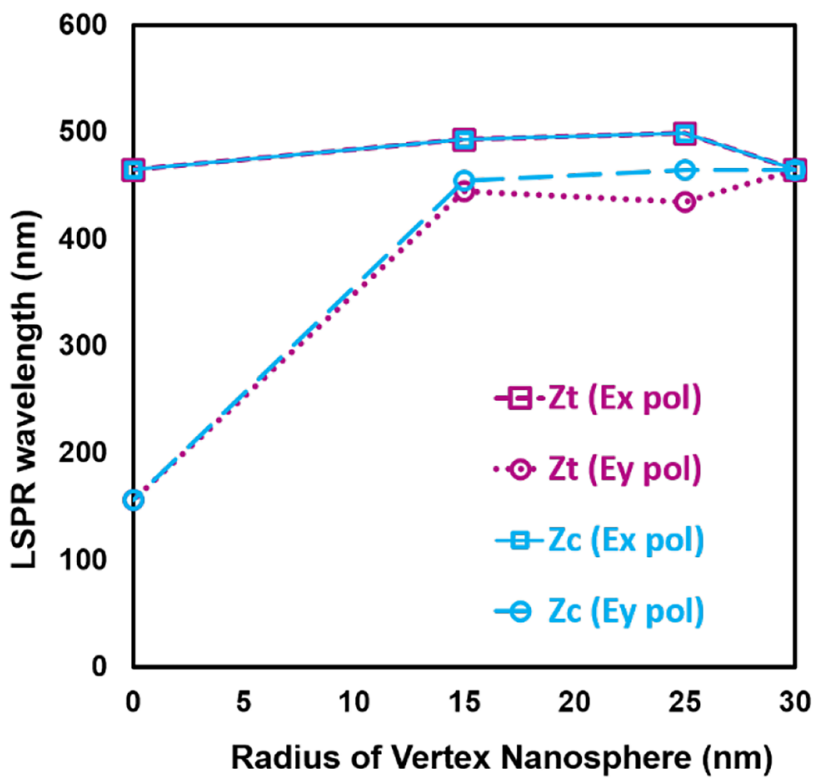

Fig. 5 The electric field enhancement (a) and LSPR wavelength (b) of the trimer series, $R_{1}=30 \mathrm{~nm}, 25 \mathrm{~nm}, 15 \mathrm{~nm}$ and $0 \mathrm{~nm}$ are calculated at $Z_{\text {centre }}$ (solid lines) and $Z_{\text {top }}$ (dotted lines) for longitudinal polarization, $E_{x}$ (square), and transverse polarization, $E_{y}$ (circle)

position of centre of vertex CS NS at the $Z_{\text {top }}$, i.e. the top surfaces of the three NSs at the same $z$-position is analysed and compared with $Z_{\text {centre }}$. In Fig. 5a and 5b, the electric field enhancement and LSPR wavelength of the trimer series are calculated at $Z_{\text {centre }}$ (solid lines) and $Z_{\text {top }}$ (dotted lines) for longitudinal, $E_{x}$ (square), and transverse, $E_{y}$ (circle), polarizations. In the case of $D_{3 \mathrm{~h}}$ symmetry configurations, there is no change in the geometry of the cluster with respect to the change in the $z$-position at the top surface; therefore, no change is observed for the LSPR mode and field enhancement. However, the geometry of the $C_{2 \mathrm{v}}$ symmetry $\left(R_{1}=15\right.$ and $25 \mathrm{~nm}$ ) is broken, i.e. the position of vertex NS is shifted when the top surface of the vertex NS is aligned with the same z-position of the two base NSs. For Ex polarization, the effect of change in $z$-position does not affect the LSPR wavelength as the plasmonic effect is caused by the bonding mode, $B_{2}$ mode, that occurs between the base NSs which lies along $x$ direction; however, a small increase in the field enhancement is observed for the $Z_{\text {top }}$. For $E_{y}$ polarization, predominant change is observed for the $C_{2 \mathrm{v}}$ symmetry due to coupling of vertex NS with base NSs along $y$ direction. The LSPR peak is blue shifted and the field enhancement shows a high difference. In the case of $Z_{\mathrm{top}}, C_{2 \mathrm{v}}$ symmetry is broken and does not obey the plasmon hybridization theory. In the case of $D_{\infty \mathrm{h}}$ symmetry, there is no change in the geometry with respect to the $Z_{\text {top }}$ as the vertex NS is absent in this linear dimer structure; therefore, no change is observed for the LSPR mode and field enhancement similar to the case of $D_{3 \mathrm{~h}}$ symmetry.

\section{Conclusions}

In this paper, the SERS characteristics for the transformation of trimer arrangement of silver@ silica CS NS cluster to dimer arrangement are numerically investigated for the first time and correlated with the plasmon modes for understanding the background physical concepts. The e-field localization corresponding to the dipolar orientation of the plasmon modes is clearly evident by the hot spot generation as represented in the spatial map. The $D_{3 \mathrm{~h}}$ symmetry exhibits high enhancement with degenerative nature. A polarization selective decrement is observed as the degeneracy in the plasmon dipolar mode is lifted for the $C_{2 \mathrm{v}}$ and $D_{\infty \mathrm{h}}$ symmetry. The optical behaviour of different series is shaped by the structure of the plasmon modes. The origin of the plasmonic behaviour of the dimer is observed from plasmon mode evolution from trimer to dimer. The effect of $z$-position on centre of vertex NS shows the significance of arrangement of NSs on symmetry breaking. This study paves way for designing SERS substrate with complex clusters such as tetramers and pentamers to study their SERS behaviour by breaking their symmetry into different geometrical configurations for polarization selective light coupling.

Author Contribution A.N. conceived of the idea, conducted theoretical and computational studies, and prepared the draft of the paper. P.C. and H.S. helped in computation and analysis on the results. A.P.P and H.I. cooperatively supervised the research. All authors participated in preparation of the final version of the manuscript. 
Availability of Data All data included in this paper are available from the corresponding author upon reasonable request.

Code Availability All the codes used to generate the data included in this paper are available from the corresponding author upon reasonable request.

\section{Declarations}

Ethics Approval Not applicable.

Consent to Participate Not applicable.

Consent for Publication Not applicable.

Conflict of Interest The authors declare no competing interests.

\section{References}

1. Chamanzar M, Xia Z, Yegnanarayanan S, Adibi A (2013) Opt Express 21:32086

2. Prezgot D, Bottomley A, Coyle JP, Ianoul A (2017) J Phys Chem Lett 8:5555

3. Unser S, Bruzas I, He J, Sagle L (2015) Sensors 15:15684

4. Bigness A, Montgomery J (2008) Materials 11:672

5. Ozhikandathil J, Packirisamy M (2014) Sensors 14:10497

6. Truong NTN, Kim CD, Reddy VRM, Thai VH, Jeon HJ, Park C (2020) J Mater Sci Mater Electron 31:22957

7. Li F, Wu S, Zhang L, Li Z (2017) J Mater Sci Mater Electron 28:16233
8. Jain P, Shokeen P, Arun P (2016) J Mater Sci Mater Electron 27:5107

9. Chen X, Yan L (2017) J Mater Sci Mater Electron 28:13698

10. Senthilkumar N, Arulraj A, Nandhakumar E, Ganapathy M, Vimalan M, Potheher IV (2018) J Mater Sci Mater Electron 29:12744

11. Magdy C, Issam F, Amir P, Adel S, Elgohary S (2020) MOJABB 4:86

12. Lee D, Yoon S (2016) J Phys Chem C 120:20642

13. Goel R, Awasthi V, Rai P et al (2021) Design of Polarization Independent SERS Substrate with Raman Gain Evaluated Using Purcell Factor. Plasmonics 16:1365-1373. https://doi.org/10. 1007/s11468-021-01410-Z

14. Goel R, Awasthi V, Rai P, Dubey SK (2021) Plasmonics 16:107

15. Campione S, Adams SM, Ragan R, Capolino F (2013) Opt Express 21:357

16. Wang AX, Kong X (2015) Materials 8:3024

17. Sio A, Shell C, Plasmon ND, Shanthil M, Thomas R, Swathi R, George Thomas K (2012) J Phys Chem Lett 3:1459

18. Ma YW, Wu ZW, Zhang LH, Zhang J, Jian GS, Pan S (2013) Plasmonics 8:1351

19. Yan B, Boriskina SV, Reinhard M (2011) J Phys Chem C 115:4578

20. Calamak S, Ulubayram K (2019) J Mater Sci Mater Electron 30:10013

21. Nagarajan A, Priya A, Chelliah P, Satoh H (2019) Appl Surf Sci 495:143457

22. Dhawan A, Norton SJ, Gerhold MD (2009) Opt Express 17:1536

23. Oskooi AF, Roundy D, Ibanescu M, Bermel P, Joannopoulos JD, Johnson SG (2010) Phys Commun 181:687

24. Djuris AB, Elazar JM, Majewski ML (1998) Appl Opt 37:5271

Publisher's Note Springer Nature remains neutral with regard to jurisdictional claims in published maps and institutional affiliations. 